\title{
Necrotizing Fasciitis: A Surgical and Medical Emergency
}

\author{
Nissar Shaikh ${ }^{1 *}$, Jamila Khawaiter ${ }^{2}$, Hassan Al-Thani ${ }^{3}$ \\ ${ }^{1}$ Consultant Surgical Intensive Care Unit, Hamad Medical Corporation, Doha, Qatar \\ ${ }^{2}$ Infectious Disease Section, Department of Medicine, Hamad Medical Corporation, Doha, Qatar \\ ${ }^{3}$ Ead General, Vascular and Trauma Surgery, Hamad Medical Corporation, Doha, Qatar \\ Email: *smaheboob@hmc.org.qa
}

Received August 5, 2012; revised September 3, 2012; accepted September 12, 2012

\begin{abstract}
Necrotizing fasciitis (NF) is still remained a dreaded disease with high morbidity and mortality due to rapidly progressive necrotizing infection. Objective: Review recent available literature on necrotizing fasciitis (NF) and compare it with our publications. Data and Source: Local and international published literature on NF from early nineties to 2012; Midline and pub Med literature search using the term "necrotizing fasciitis". Data Synthesis: NF is more common in male patient, it is frequently polymicrobial. Common site of NF is the extremities. NF is classified according to the microbes. Common co-morbid condition associated with NF is diabetes mellitus. Minor trauma and surgery are the major etiological risk factors for NF. There seems to be significant correlation between the use of non steroidal anti-inflammatory medication (NSAID) and NF. Severe pain disproportionate to injury is the presenting symptom. Laboratory risk indicators for necrotizing fasciitis (LRINEC) score will diagnose NF early in emergency clinics. Tissue biopsy is the gold standard for the diagnosis of NF. Pathophysiology of NF is rapid horizontal spread of infection with fascial necrosis and secondary vertical involvement of skin. More than 90\% of NF patients need intensive care therapy. Early and bold debridement in combination with brave organ supportive intensive care will have better outcome of NF patients. Conclusion: High index of suspicion and knowledge is essential for early diagnosis of NF. Finger test and LRINEC score may help in early diagnosis. Early debridement, proper antibiotics and organ supportive intensive care will improve morbidity and mortality of NF patients.
\end{abstract}

Keywords: Necrotizing Fasciitis; Surgical; Medical Emergency

\section{Introduction}

Necrotizing fasciitis (NF) is a rapidly progressing, inflammatory infection of the fascia with the secondary involvement of skin, subcutaneous tissues and muscle [1]. NF is a limb and life threatening surgical emergency; we added the term medical emergency as it requires emergent and aggressive medical care as most of these patients are managed in the intensive care unit [2]. Various terminologies are used describe necrotizing fasciitis (NF) such as hospital gangrene, streptococcal gangrene, acute dermal gangrene, Fournier's gangrene suppurative fasciitis, synergistic necrotizing cellulites. NF is a severe form of soft tissue infection.

$\mathrm{NF}$ is recognized as dreaded disease from the days of Hippocrates; Fournier in late eighteen century documented necrotizing infection of the perineal and genital area; which is still known as Fournier' gangrene. Wilson in 1952 gave the term 'necrotizing fasciitis' to describe the disease and it is the preferred terminology in these days, as it describes the most consistent and key features of the disease; the fascial necrosis. Necrosis means death

${ }^{*}$ Corresponding author. of a portion of the tissue and fascia is fibrous tissue that encloses muscle [3].

NF in last century occurred sporadically mainly during the war time and it was monobacterial; but recently its occurrence in civilian population is on rise and it's mainly polymicrobial and methicilin resistant staphylococcal infection (MRSA) [4].

High morbidity and mortality associated with NF makes it an emergency; early debridement will have a favourable outcome. Hence, it is a surgical emergency. More than $90 \%$ of NF patients will also need intensive care and organ supportive therapy; that makes NF a medical emergency. Up to $46 \%$ of NF patients may need limb amputation or disarticulation [2]. All above explanations dictate that the NF a limb and life threatening surgical as well as medical emergency.

\section{Epidemiology}

One has to be careful about the difficulties to record actual incidence of NF due the confusion by various eponyms of necrotizing fasciitis. In USA the NF occurs in 4.3 per 100,000 of the population [2]. In UK it is re- 
ported that 500 new case of NF per year [5]. In our study 94 patients were admitted to the surgical intensive care unit over a period of 10 years [6]. NF commonly occurs in male with male:female ratio of $3: 1$, it more frequently affects the extremities [6]. In our study 54\% of the patients had NF of the extremities. Anaya et al. had 58.7\% of their patients had NF of the extremities [7]. NF involving perineum and genitalia occurs in 20\%; chest, flanks, shoulder as well as hip and gluteal area are involved with NF in $8.5 \%$ of the patients. Fortunately abdominal and cervical necrotizing fasciitis are rare $\mathbf{5 . 3 \%}$ and $2.1 \%$, respectively) [8]. NF is rare in pediatric and neonatal age; rarely reported NF affecting scalp [9].

\section{Classification}

Necrotizing fasciitis is classified according to the area involved and microbes causing necrotizing fasciitis. When the NF occurs without any initiating events it is called as idiopathic necrotizing fasciitis [9]. According to the body area involved with the necrotizing fasciitis; it is called: 1) Cervical necrotizing fasciitis, when area involves head and neck; 2) Abdominal necrotizing fasciitis (Meleney’s gangrene); 3) Fournier's gangrene: Perineum and genitalia necrotizing fasciitis.

According to microorganism causing necrotizing fasciitis; it is classified into four groups.

\subsection{Type I Necrotizing Fasciitis}

This type of NF is a polymicrobial, often bowel flora derived and synergistic infection; tissue culture will show a mixed growth of anaerobes and aerobes. It is common type of NF; frequently occurs in patients with diabetes mellitus. Type $1 \mathrm{NF}$ common to occur in perineum and trunk of the body [6-8].

\subsection{Type II Necrotizing Fasciitis}

It is a monobacterial, skin or throat derived infection, often the causative organism will be either Group A/B hemolytic streptococci alone or in combination with the staphylococci. Even staphylococci alone can also cause this type of NF [6].

Type 2 NF commonly occurs in young and immunecompetent patients. Type NF due to MRSA is increasing. Frequently involved areas of the body with this infection are the extremities. Type 2 NF occurred in $43 \%$ of our patients. Usually these patients will have toxic shock syndrome and multiple organ dysfunctions [6-8].

\subsection{Type III Necrotizing Fasciitis}

This is the fulminant form of NF. It is caused by gram negative bacteria, commonly by marine-related organisms. Typically occurs after punctured wound from fish or cut injury or an insect bite exposed to the sea water. It is a hyper acute infection, causes septic shock and MODS within 12 to 24 hours of injury; if not detected early it will leads to $100 \%$ mortality [10]. In a study from Hong Kong showed $83 \%$ of their totals NF were type 3 [2]. We had reported a case of type 3 NF of the forearm, caused by the Aeromonas sabori; patient developed septic shock with MODS with in 12 hour of injury [11].

\subsection{Type IV Necrotizing Fasciitis}

This type of NF occurs in immunocompromised or severe trauma patients. It is caused by fungal infection. It can rapidly spread and leads to severe NF. It caused by candida or Mucor and Rhizopus species (Table 1).

\section{Risk Factor}

Diabetes mellitus is one of the commonest risk factor for the NF; Chueng et al. reported that up to $57 \%$ of their patients had diabetes mellitus [2]. In our study 53\% of the patients were diabetic; we also reported other risk factors for NF; Hypertensive disorders (25\% Patients), cardiac diseases (15\%), chronic airway diseases (5.3\%) and brachial asthma in $2.1 \%$ of the NF patients [6].

There seems to be significant correlation between development of NF and use of non-steroidal anti-inflammatory (NSAID) drugs. Souyri et al. claimed a significant association the NF and use of NSAID with OR = 31.4; 95\% CI, 6.4 - 15.3 [12]. In our study 50\% of NF patients had received NSAID before presenting to the hospital [6]. NSAID suppresses cytoprotective effect of prostaglandins; and as these medications reduces pain, while the disease process is continued resulting in late presentation to the hospital. We also found that minor trauma (11.7\%), surgical procedure (10.6\%) and insect bite were etiological risk factors for the development of NF [6] (Table 2).

\section{Clinical Presentation}

NF can affect any region or part of the human body. The major clinical suspicion of NF should arise when patient complains of severe pain disproportionate to the local injury.

Common presenting sign and symptoms of NF are severe pain, swelling and redness in $78 \%-100 \%$ of these patients. Fever in $86 \%$ of NF patients, 33\% will be presenting as hypotension. Forty percent of NF patients will have local crepitus and blistering. 24\% of them had local hypoesthesia or anesthesia and 23\% of NF patients will be disoriented at the presentation [13]. Tang et al. reported all of their NF patients had severe pain at the site of NF, $58 \%$ had tenderness and swelling whereas erythema was present in $50 \%$ of patients. Interesting but significant finding in Tang et al. study was that $79 \%$ of 
Table 1. Types of necrotizing fasciitis (NF).

\begin{tabular}{cc}
\hline Type I & Polybacterial and common \\
\hline Type II & Monobacterial/fulminant \\
Type III & Marine bacteria/fulminant \\
Type IV & Fungal \\
\hline
\end{tabular}

Table 2. Risk factors for necrotizing fasciitis.

Age $>50$ years
Malnutrition
Intravenous drugs abuser
Diabetes
Chronic liver disease
Chronic kidney disease
Steroids
Malignancy
Obesity
Peripheral vascular disease
Minor trauma
Surgical procedure
Chrect bite; Hypertensive disorders; Cardiac diseases;
Chronic airway disease.

the NF patients presented to the hospital in toxic shock syndrome [14].

To summarize the initial presentation of NF, all patients will have severe pain in the affected area or whole limb. There may be signs of trivial injury or skin opening. Patient will be dehydrated and looks sicker. If patient present within 3 - 4 days, the area may be swollen, warm, rash and affected limb or area might have large, dark colored marks which progress into the blisters, than Wounds becomes necrotic. Wang et al. graded the dermatological manifestations of NF into 3 stages depending on early or late presentation of NF patients [15] (Table 3).

\section{Diagnosis}

Diagnosis is the important but difficult aspect of the management of NF; initially patients' manifestations are benign and confusing and no any initial specific sign is not diagnostic of NF. It is a common mistake to diagnosis NF as cellulitis, which will dilute the patient care. Patient with NF looks sicker on presentation to the emergency department and few basic blood investigations (complete blood count, serum electrolytes and acute phase proteins) will be done on presentation.

Wong et al. depending on hypothesis that these basic above mentioned profiles may help in the early diagnosis of NF, did a comparative study between cellulitis and NF; designed the laboratory risk indicatotors for necrotizing fasciitis (LRINEC) score. It is a numerical score (Table 4) with a total of 13. If LRINEC score of 6 or greater has positive predicative value for NF of $92 \%$ and negative predicative value of 96\%. A LRINEC score 8 and above will have a stronger positive predicative value with a specificity of 95\% (Table 4) [16].

The beauty of the LRINEC score is its easy to calculate and parameters are often readily available in the emergency department; which will facilitate early diagnosis. In our study we found that almost all our NF patients had fever and leucocytosis on presentation to the emergency department [6]. The tissue oxygen monitoring with the

Table 3. Stages of necrotizing fasciitis.

\begin{tabular}{ccc}
\hline Stage1/Early & Stage2/Intermediate & Stage3/Late \\
\hline Erythema & Blisters & Crepitus \\
Swelling & Bullae & Anesthesia \\
& Warm skin & Skin necrosis \\
\hline
\end{tabular}

Table 4. LRINEC sore.

\begin{tabular}{|c|c|}
\hline Value & Score \\
\hline \multicolumn{2}{|c|}{ C-reactive protein $(\mathrm{mg} / \mathrm{L})$} \\
\hline$<150$ & 0 \\
\hline$>150$ & 4 \\
\hline \multicolumn{2}{|l|}{ Leukocytosis } \\
\hline$<15,000$ & 0 \\
\hline $15,000-25,000$ & 1 \\
\hline$>25,000$ & 2 \\
\hline \multicolumn{2}{|l|}{ Hemoglobin (g/dL) } \\
\hline$>13.5$ & 0 \\
\hline $11-13.5$ & 1 \\
\hline$<11$ & 2 \\
\hline \multicolumn{2}{|c|}{ Serum sodium(mmol/L) } \\
\hline$>135$ & 0 \\
\hline$<135$ & 2 \\
\hline \multicolumn{2}{|c|}{ Serum creatinine (mg/dL) } \\
\hline$<1.6$ & 0 \\
\hline$>1.6$ & 2 \\
\hline \multicolumn{2}{|l|}{ Glucose (mg/dL) } \\
\hline$<180$ & 0 \\
\hline$>180$ & 1 \\
\hline
\end{tabular}


near-infrared spectroscopy for the diagnosis of the NF, looks a promising tool but required a properly designed studies to support its use [17].

Imaging studies also claimed to be useful in the diagnosis of the NF, but one should be very careful not to waste the life saving time, while waiting for the imaging studies.

Plain x-ray can show soft tissue swelling or subcutaneous gas, but it is not of any value for diagnosing NF. In a low powered, retrospective study ultrasound examination correlated with histopathology of NF. However as it is operator dependent; it reported to have missed few histological apparent NF [18]. Ultrasound guided aspiration of the Peri fascial fluid can help in isolating organism and narrow spectrum antibiotic therapy [19]. Computerized tomography (CT) scan is documented to have sensitivity of $80 \%$ in diagnosis of NF; it will show fascial edema, and thickening with or without gas formation [20].

Literature suggest Magnetic resonance imaging (MRI) is highly sensitive (90\%) but not specific (50\% - 80\%) in the diagnosis of NF. Hence MRI shows more positive NF; and MRI is not routinely available for 24 hours and in all the hospitals [21].

\section{Surgical Diagnosis}

Mainly two surgical modalities are available for the diagnosis of the necrotizing fasciitis.

Finger test: This was described by Andreasen et al. A 2-cm long incision under local anesthesia is made in the affected area, lack of fascial resistance, active bleeding and foul smelling fluid will constitute a positive finger test; which will be diagnostic of necrotizing fasciitis [22]. Biopsy/Frozen section biopsy: Majeski et al. supported the idea of frozen section biopsy; they also demonstrated that it diagnoses NF early and hence reduces the mortality. But it needs dedication and willingness of a pathologist familiar with the interpretation of NF [23].

Gold standard for detecting and diagnosing necrotizing fasciitis is biopsy either tissues taken during or after the debridement [2].

\section{Pathophysiology}

Necrotizing fasciitis pathophysiology is hall mark by trivial injury but rapidly progressive necrotizing infection of the superficial fascia. It is essential to understand the pathophysiology of NF it discerning the presentation of the dreaded disease.

In necrotizing fasciitis the primary site of infection is superficial fascia, than the infection rapidly spread through the fasical planes (horizontal spread) without involvement of skin. This horizontal spread start with initial trivial injury, the bacteria get lodged into the su- perficial fascia, proliferate and produces hyaluronidase enzymes. This enzyme causes degradation and necrosis of the fascial layers; creates more favorable atmosphere for bacterial growth and rapid, uncontrolled spread of infection. This is the reason that patients become sicker without significant local manifestations. As the disease evolves, vertical spread of infection occur leading to the involvement of skin, subcutaneous tissue, deeper fascia and muscles [24].

Once the necrosis of the superficial fascia occurs, it causes leucocyte infiltration in the deeper fascia, dermis, thrombosis and suppuration of veins and arteries passing through the fascia. This leads to occlusion of the dermal nutrient vessels and causes progressive ischemia and gangrene of the overlying skin [24].

In type II necrotizing fasciitis the causative organism streptococcus produce a large number of virulence factors; which damages host tissues by inactivating polymorphonuclear cells. Whereas the exotoxin secreted by these bacteria enhances the virulence and accelerates progression of the infection [25].

Streptococcus and staphylococci produces surface antigen M1, M3, exotoxins A, B, C streptolysin O, and super antigens. An $\mathrm{M}$ protein increases adherence ability of these bacteria, which helps microbes in avoiding the phagocytosis. Exotoxin A and B causes loss of vascular integrity, which leads to capillary leakage and tissue edema $[23,24]$. Over all effects of all these above mentioned virulent factors cause much rapid spread of infection in type2 NF; hence these types of patients frequently present with the toxic shock syndrome and multiorgan dysfunction syndrome.

\section{Treatment}

Bold early surgical debridement, appropriate early antibiotics and brave medical organ supportive intensive care is essential for better outcome of NF patients. Early and aggressive debridement of all necrotic tissues until the tissue start to bleed is the pillar of NF management and may need more than one debridement; hence repeated wound examination is mandatory, and these patients' needs on average two or more setting of debridement [6]. Delayed or incomplete debridement will increase the morbidity and mortality, toxemia, dehydration and biochemical disorders [26].

In NF surgical debridement of necrotic tissue within 24 hours of presentation to the hospital has significantly better outcome than the delayed debridement done $6 \%$ verses 25\%) [27]. Repeated aggressive debridement decrease mortality in the NF patients [28].

NF patients wound care has to be taken meticulously. These wounds should be washed and dressed with the occlusive, adsorptive dressing with bandage two to three 
times in a day. It is recommended to use antibiotic dressing (1\% povidone iodine or silver sulfadiazine) [29].

Perineal, perianal or scrotal wounds requires special considerations, it may be necessary to perform diverting colostomy, cystostomy or both to facilitate the granulation formation and wound hygiene [29].

Along with the aggressive surgical debridement, proper medical therapy is the essential part of management of NF patients. All NF patients with even mild multi-organ dysfunction syndrome (MODS) should be admitted to the intensive care unit. Antibiotics therapy should be started as soon as blood samples were taken for microbiological work-up. Common type of NF is polymicrobial; initial antibiotic regimen should include agents effective against gram positive cocci, gram negative bacilli and anaerobes. Initial antibiotic therapy will have two antimicrobial agents. One of these should be clindamycin [23]. Clindamycin has few added advantage in these patients, the efficacy of clindamycin is independent of inoculum size or bacterial growth, it is a potent suppressor of the bacterial toxins, its sub-inhibitory concentration also facilitates phagocytosis, it reduces the synthesis of penicillin binding proteins, have a longer post antibiotic and immuno-modulating effect (as it inhibits the synthesis of tumor necrosis factor) [30].

In our decade long experience in the treatment of NF, most of theses patients received two antibiotics, and commonly used antibiotic were Tazocin $^{\circledR}$ (73\%) and clindamycin (64\%) [6]. As soon as microbiological results were available we should narrow the antibiotic spectrum (Table 5).

Intravenous immunoglobulins (IVIG) are useful in patients with NF with toxic shock syndrome. As they $n$ tralizes the super antigens; $\beta$ hemolytic streptococcal activity on cytokine release and reduces the plasma tumor necrosis factor and interleukin 6 levels [27]. Kaul et $a l$. in their case controlled study demonstrated that IVIG use in toxic shock syndrome yielded 67\% survival when compared to the control group 34\% [31].

Organ supportive therapy and resuscitation is a con-

Table 5. Antibiotics use in necrotizing faciitis.

\begin{tabular}{ccc}
\hline Antibiotics & Count & Percentage (\%) \\
\hline Tazocin & 69 & 73.4 \\
Clindamycin & 60 & 63.8 \\
Metronidazole & 21 & 22.3 \\
Amikacin & 13 & 13.8 \\
Ceftriaxone & 11 & 11.7 \\
Gentamycin & 4 & 4.3 \\
Vancomycin & 3 & 3.2 \\
\hline
\end{tabular}

tinuous process in NF patients. As high a $79 \%$ of these patients will require invasive ventilation and $70 \%$ will be on vasopressors [32]. NF patients may progress to the other organ dysfunction. NF patients have a large volume of extracellular fluid sequestration as well as dehydration; in our study we reported that NF patients required average 5 liters of fluid resuscitation in initial 24 hours of admission. These patients need blood and blood products as result of severe sepsis and surgical interventions. In our series NF patients received at least 2 units pack red blood cells, 4 units of fresh frozen plasma and 5 units of the platelet concentrate within 24 hours of admission [6].

Adequate nutrition support is important for the better outcome of NF patients. Early enteral feeding will have obvious benefits, and in acute phase of NF it is recommended to supplement twice of basic caloric requirement [22,33].

Use of hyperbaric oxygen therapy (HBOT) in NF patients remains a controversial issue. It is advised that HBOT should be given in the post-debridement period. It should be taken care that transfer of NF to a hospital equipped with the HBOT should not delay the emergency debridement surgery. There are no large, randomized controlled study trails in support of beneficial effects of HBOT in NF patients. HBOT increases the oxygen saturation in the infected tissues by 1000 folds; has a bacteristatic effect, improves polmorphonuclear function, stops spread of the disease processes and enhances the wound healing. The standard HBOT is 2 to $2.5 \mathrm{~atm}$ for 90 to 120 minutes twice daily [34].

Once these NF recovered from emergent phase may require reconstructive surgery. NF patients often have a massive loss of skin and subcutaneous tissues. Type of reconstructive surgery is dictated by the amount of soft tissue or skin is loosed. The reconstructive surgeries can be skin graft, rotational flap surgeries or a biological mesh repair [35].

The tropical negative pressure therapy has been reported to improve wound healing by removing exudates, covering wound securely and by stimulating angiogenesis [29].

\section{Complications}

NF can leads to various complications. These patients may have septic shock or toxic shock syndrome and multi-organ dysfunction syndrome (MODS); upto 79\% of these patients requires invasive ventilation and $70 \%$ will be on vasopressors [31]. If infection is not controlled and progressive inspite of debridement may need amputation; the percentage and rate of amputation varies. The risk factors for amputation are myonecrosis, unremitting shock, concurrent vascular insufficiencies, rapidly progressing 
infection and large area of soft tissue necrosis [2].

A small Turkish study reported $40 \%$ of NF patients had above or below knee amputation [36]. According to Mouzopoulos et al. 23\% of NF patients required amputation or limb disarticulation [37]. We published few unusual complications; $6.4 \%$ of our patients had ventricular tachycardia, 5.3\% had compartment syndrome, one patient had tension pneumothorax due to the raptured post type II necrotizing fasciitis pneumocel [6] and one patient had acute myocardial infarction [38].

\section{Prognosis}

Necrotizing fasciitis has a mortality of $20 \%$ to $75 \%$ [2]. Necrotizing fasciitis in pediatric and neonatal age has higher mortality of $52 \%$ and $72 \%$ respectively [9]. Most important risk for the mortality is delayed surgical intervention; if the surgical intervention delayed for 24 hours or more causes significant increase in mortality; other risk factors for increased mortality are older age, diabetes mellitus and two or more co-morbid disease [2-26]. In our study there was no significant difference in mortality of diabetic and non-diabetic NF patients [39]. Ogilvie et al. reported other factors leukocytosis of more than $30 \times$ $10^{9}$, hyperkalemia, increased partial thromboplastin time and low $\mathrm{PH}$ on admission will increased mortality NF patients [40]. Hsiao et al. found that Vibrio and Aeromonas infection, hypotension on admission and malignancy were associated with increased mortality in NF patients while presence of hemorrhagic bullae was associated with reduced mortality [41]. Mortality according to the body area involved; necrotizing fasciitis of chest, axilla, and flanks as well gluteal region has highest as mortality (25\%), Cervical NF has a mortality of $20 \%$ and NF involving perineum and genitialias has lowest mortality of $10 \%$ [8]. Mortality according to the type of NF varies, Mortality of type I NF patients depends mainly on associated co-morbid conditions; type II NF has a mortality of 32\% depending on presentation, type III NF has a mortality of $30 \%$ to $40 \%$ and type IV NF has mortality of more than $40 \%$ [42].

\section{Prevention}

According to the center for disease control and prevention; following are the recommendation for prevention and spread of NF [43].

1) Good hand washes after coughing, sneezing and before/after the patient care.

2) Keeping skin intact.

3) Patients with an infected wound with fever should seek early medical care.

4) Patients with sore throat should be seen by physician.

\section{Conclusion}

Necrotizing fasciitis is a life and limb threatening rapidly progressive necrotizing infection. It is commonly polymicrobial and MRSA necrotizing fasciitis is on rise. NF more frequently affects male patients. NSAID use is increasingly blamed for NF. It is classified into 4 types depending on causative microbes, type II and III are fulminant forms of NF. All NF patients will present with severe pain which will be disproportionate to the local manifestations. Dermatological manifestations of NF can be graded into 3 stages depending on early or late presentation. LRINF score, Finger test and frozen section biopsy will help in early diagnosis of NF. NF pathophysiology is divided into two phases; initial early rapid horizontal spread of infection with fascial necrosis and then the secondary vertical involvement of overlying skin. Rarely NF can complicate into acute myocardial infarction and tension pneumothorax. Early bold debridement, earlier proper antibiotics in combination with organ supportive intensive care therapy will improve NF patients' outcome. Hence we labeled NF as surgical as well as medical emergency.

\section{REFERENCES}

[1] M. S. Morgan, "Diagnosis and Management of Necrotizing Fasciitis,” Journal of Hospital Infection, Vol. 75, No. 4, 2010, pp. 249-257. doi:10.1016/j.jhin.2010.01.028

[2] J. P. Y. Cheung, B. Fung, W. M. Tang and W. Y. Ip, “A Review of Necrotizing Fasciitis in the Extremities," Hong Kong Medical Journal, Vol. 15, No. 1, 2009, pp. 44-52.

[3] B. Wilson, "Necrotizing Fasciitis," The American Journal of Surgery, Vol. 18, No. 4, 1952, pp. 416-431.

[4] Miller et al., "Necrotizing Fasciitis Caused by Community Associated Methicilin Resistant Staphylococcal Infection in LA," The New England Journal of Medicine, Vol. 352, No. 14, 2005, pp. 1445-1453. doi:10.1056/NEJMoa042683

[5] H. Saiidy, P. Matteucci, P. R. W. Stanley and N. B. Hart, "Necrotizing Fasciitis," British Medical Journal, Vol. 330, No. 7495, 2005, pp. 830-833.

[6] N. Shaikh, "Necrotizing Fasciitis, a Decade of Surgical Intensive Care Experience," Indian Journal of Critical Care Medicine, Vol. 10, No. 4, 2006, pp. 225-229.

[7] D. A. Anaya, K. McMahon, A. B. Nathens, S. R. Sullivan, H. Foy and E. Bugler, "Predictors of Mortality and Limb Loss in Necrotizing Soft Tissue Infections," Archives of Surgery, Vol. 140, No. 2, 2005, pp. 151-157. doi:10.1001/archsurg.140.2.151

[8] N. Shaikh and A. Rashid, "Regional Necrotizing Fasciitis and Its Outcome,” Qatar Medical Journal, Vol. 17, No. 1, 2008, pp. 24-27.

[9] S. Ozturk, F. Zur, Y. Karslioglu and M. Sengezer, "Idiopathic Neonatal Necrotizing Fasciitis of Scalp. A Case Report,” European Journal of Plastic Surgery, Vol. 28, 
No. 5, 2005, pp. 368-370. doi:10.1007/s00238-005-0801-8

[10] K. H. Goodell, M. R. Jordan, R. Graham, et al., "Rapidly Advancing NF Caused by Phytobacterium (Vibrio). A Hyper Aggressive Variant," Critical Care Medicine, Vol. 32, No. 1, 2004, pp. 278-281. doi:10.1097/01.CCM.0000104920.01254.82

[11] N. Shaikh, M. A. kattern and A. Ahmed, "Necrotizing Fasciitis Caused by Aeromonas sabori," The Middle East Journal of Emergency Medicine, Vol. 7, 2007, pp. 30-32.

[12] C. Souyri, P. Oliver, S. Grolleau and M. Lapeyre-Mastre, "French Network of Pharmaco-Vigilance Centers. Severe soft Tissue Infections and Non-Steroidal Anti-Inflammmatory Drugs," Clinical and Experimental Dermatology, Vol. 33, No. 3, 2008, pp. 249-255. doi:10.1111/j.1365-2230.2007.02652.x

[13] I. Brook and E. H. Frazier, "Clinical and Microbiological Features of Necrotizing Fasciitis," Journal of Clinical Microbiology, Vol. 33, No. 9, 1995, pp. 2382-2387.

[14] W. M. Tang, P. L. Ho, K. K. Fung, K. Y. Yen and J. C. Leong, "Necrotizing Fasciitis of a Limb," Journal of Bone \& Joint Surgery, British Volume, Vol. 83, No. 5, 2001, pp. 709-714. doi:10.1302/0301-620X.83B5.10987

[15] Y. S. Wang, et al., "Staging of Necrotizing Fasciitis Based on the Evolving Cutaneous Features," International Journal of Dermatology, Vol. 46, No. 10, 2007, pp. 1036-1040. doi:10.1111/j.1365-4632.2007.03201.X

[16] C. H. Wong, L. W. Khin, K. S. Heng, et al., ”The LRINEC (Laboratory Risk Indicatotors for Necrotizing Fasciitis) Score. A Tool for Distinguish Necrotizing Fasciitis from Cellulites," Critical Care Medicine, Vol. 32, 2004, pp. 1535-1540. doi:10.1097/01.CCM.0000129486.35458.7D

[17] T. L. Wong and C. L. Hung, "Role of Tissue Oxygen Monitoring in Diagnosing Necrotizing Fasciitis of Lower Limbs,” Annals of Emergency Medicine, Vol. 44, No. 3, 2004, pp. 222-228. doi:10.1016/j.annemergmed.2004.03.022

[18] G. C. Parenti, C. Marri, G. Calandra, C. Morisi and W. Zabberoni, "Necrotizing Infection of Soft Tissue. Role of Diagnostic Imaging and Review of Literature," Radiologia Medica (Torino), Vol. 99, 2000, pp. 334-339.

[19] H. C. Chao, M. S. Kong and T. Y. Lin, "Diagnosis of Necrotizing Fasciitis in Children,” Journal of Ultrasound in Medicine, Vol. 18, 1999, No. 277-278.

[20] M. G. Wysoki, et al., "Necrotizing Fasciitis: CT Characteristics,” Radiology, Vol. 203, No. 3, 1997, pp. 859-863.

[21] M. R. Schmid, et al., "Differentiation of Necrotizing Fasciitis from Cellulitis, Using MR Imaging," American Journal of Roentgenology, Vol. 170, 1998, pp. 615-620.

[22] T. J. Andreasen, et al., "Massive Soft Tissue Injury: Diagnosis and Management of Necrotizing Fasciitis and Purpura Fulminans," Plastic and Reconstructive Surgery, Vol. 107, 2001, pp. 1025-1035. doi:10.1097/00006534-200104010-00019

[23] J. Majeski and E. Majeski, "Necrotizing Fasciitis: Improved Survival with Early Recognition by Tissue Biopsy and Aggressive Debridement,” Southern Medical Journal,
Vol. 90, No. 11, 1997, pp. 1065-1068. doi:10.1097/00007611-199711000-00001

[24] D. V. Seal, "Necrotizing Fasciitis," Current Opinion in Infectious Diseases, Vol. 14, No. 2, 2001, pp. 127-132. doi:10.1097/00001432-200104000-00003

[25] B. Sarani, et al., "Necrotizing Fasciitis: Current Concepts and Review of the Literature," Journal of the American College of Surgeons, Vol. 208, No. 2, 2009, pp. 279-288. doi:10.1016/j.jamcollsurg.2008.10.032

[26] U. I. Ugboko, E. Assam, F. O. Oginni and A. O. Amole, "Necrotizing Fasciitis of Head and Neck: A Review of Literature,” Nigerian Medical Journal, Vol. 10, No. 1, 2001, pp. 6-10.

[27] S. T. Lille, T. T. Sato, L. H. Engrave, H. Foy and G. J. Jurkovich, "Necrotizing Soft Tissue Infection: Obstacles in Diagnosis," Journal of the American College of Surgeons, Vol. 182, 1996, pp. 7-11.

[28] B. D. Bilton, G. B. Zibari, R. W. McMillan, D. F. Aultman, G. Dunn and J. C. McDonald, “Aggressive Surgical Management of Necrotizing Fasciitis Serves to Decrease Mortality. A Retrospective Study,” Annals of Surgery, Vol. 64, No. 5, 1998, pp. 397-400.

[29] Z. Roje, Z. Roje, D. Matic, D. Librenjak, S. Dokuzovic, J. Varvodic, "Necrotizing Fasciitis: Literature Review of Contemporary Strategies for Diagnosis and Management: Three Case Reports: Tarso, Abdominal Wall, Upper and Lower Limbs," World Journal of Emergency Surgery, Vol. 6, 2011, p. 46. doi:10.1186/1749-7922-6-46

[30] R. F. Edlich, K. L. Winters, C. R. Woodard, L. D. Britt and W. B. Long, "Massive Soft Tissue Infections: Necrotizing Fasciitis and Purpura Fulminans," Journal of Long-Term Effects of Medical Implants, Vol. 15, No. 1, 2005, pp. 57-65. doi:10.1615/JLongTermEffMedImplants.v15.i1.70

[31] R. Kaul, A. McGeer, et al., "Intravenous Immunoglobulin Therapy for Streptococcal Toxic Shock Syndrome-A Comparative Observational Study," Clinical Infectious Diseases, Vol. 28, No. 4, 1999, pp. 800-807. doi:10.1086/515199

[32] M. Hassell, P. Fagan, P. Carson and B. J. Currie, “Streptococcal Pyogens Necrotizing Fasciitis from Diverse Strains in Tropical Northern Australia. Comparison with the Literature,” BMC Infectious Diseases, Vol. 4, No. 1, 2004, p. 60. doi:10.1186/1471-2334-4-60

[33] B. J. Childers, L. D. Potyondy, R. Nachreiner, et al., "Necrotizing Fasciitis: A Fourteen Years Retrospective Study of 163 Consecutive Patients," The American Journal of Surgery, Vol. 68, No. 2, 2002, pp. 109-116.

[34] L. K. Weaver, "Hyperbaric Oxygen Therapy in Critically Ill,” Critical Care Medicine, Vol. 39, 2011, pp. 17841791. doi:10.1097/CCM.0b013e31821858d1

[35] S. J. Mathes, P. M. Steinwald, R. D. Foster, W. Y. Hoffman and J. P. Anthony, "Complex Abdominal Wall Reconstruction: A Comparison of Flap and Mesh Closure," Annals of Surgery, Vol. 232, No. 4, 2000, pp. 586-596. doi:10.1097/00000658-200010000-00014

[36] M. Ozalay, G. Ozkoc, S. Akpinar, M. A. Hersekli and R. N. Tandogan, "Necrotizing Soft Tissue Infection of a 
Limb. Clinical Presentationand Factors Related to Mortality," Foot and Ankle International, Vol. 27, No. 8, 2006, pp. 598-605.

[37] G. Mouzopoulos, M. Stamatakos, S. Tzurbak, et al., "Lower Extremity Infections by Vibrio Vulrificus," Chirugia, Vol. 96, 2006, pp. 868-605.

[38] A. El-Menyar and N. Shaikh, "Necrotizing Fasciitis Causing Severe Myocardial Dysfunction with ST-Segment Elevation in a Young Man," American Journal of Emergency Medicine, Vol. 28, No. 2, 2010, pp. 260 e3260 e5.

[39] N. Shaikh, "Necrotizing Fasciitis and Diabetes Mellitus," International Journal of Diabetes in Developing Countries, Vol. 27, 2007, pp. 15-17.

[40] C. M. Ogilvie and T. Miclau, "Necrotizing Soft Tissue
Infections of the Extremities and Back," Clinical Orthopaedics and Related Research, Vol. 447, 2006, pp. 179186. doi:10.1097/01.blo.0000218734.46376.89

[41] C. T. Hsiao, H. H. Weng, Y. D. Yuan, C. T. Chen and I. C. Chen, "Predictors of Mortality in Patients with Necrotizing Fasciitis," The American Journal of Emergency Medicine, Vol. 26, No. 2, 2008, pp. 170-175. doi:10.1016/j.ajem.2007.04.023

[42] www.hkma.org/english/cme/onlinecme/cme201206set.ht $\mathrm{m}$

[43] Group A Streptococcal (GAS) Disease, Center for Disease Control and Prevention.

Http/www.cdc.gov/ncidod/dbmd/diseaseinf/groupastrepto cocal.g.htm 